\title{
Keeping 53BP1 out of focus in mitosis
}

\author{
Cell Research (2014) 24:781-782. doi:10.1038/cr.2014.62; published online 9 May 2014
}

\section{A recent study published in Science reveals the mechanism and biological importance of DNA damage response abrogation in mitotic cells.}

For many years, much research has focused on understanding how cells maintain genome integrity despite DNA being constantly challenged by factors of both endogenous and exogenous nature. DNA double-strand breaks (DSBs) are the most deleterious DNA lesions, and if left unrepaired or repaired incorrectly, a single DSB can trigger genome instability or even cell death [1]. Therefore, any DSB has to be recognized and repaired by processes encompassed within the DNA damage response (DDR). Notably, while the ends of mammalian linear chromosomes naturally resemble DSBs, their structure and association with the so-called "Shelterin" complex normally makes them invisible to the DDR [2].

As soon as a DSB is formed, it is sensed and directly bound by the Ku70Ku80 and/or MRE11-NBS1-RAD50 protein complexes, which recruit and activate the DDR kinases DNA-PKcs and ATM, respectively. The first steps in the DDR to DSBs are followed by cascades of events involving protein posttranslational modifications (PTMs) and formation of large protein assemblies at DSB sites known as ionizing radiationinduced foci (IRIF) [3]. Protein phosphorylation and ubiquitylation are at the heart of these signaling processes [3]. For example, following recruitment of the DDR mediator protein MDC1 to the phospho-epitope created by ATM and DNA-PKcs on variant histone H2AX, MDC1 is itself phosphorylated by ATM on multiple serines and threonines [4]. MDC1 phosphorylation on a group of threonines near its $\mathrm{N}$-terminus and conforming to the consensus TQXF generates binding sites for the FHA domain of E3-ubiquitin ligase RNF8 $[5,6]$. Together with the E2-conjugating enzyme UBC13, RNF8, and another E3 ligase, RNF168, trigger formation of mainly lysine 63-linked ubiquitin adducts in DSB-proximal chromatin, promoting recruitment of downstream factors necessary for DNA repair, such as the RAP80-Abraxas-BRCA1 complex and 53BP1 [3].

Significantly, the full DDR happens only in interphase cells, whereas if mitotic cells sustain DSBs, the process appears to be blocked at the stage of RNF8 recruitment, resulting in IRIF devoid of detectable ubiquitin conjugates [7]. Consequently, 53BP1 and BRCA1 are not recruited to IRIF during mitosis. Even more strikingly, although RNF8 and RNF168 are associated with mitotic IRIF in anaphase, hyperphosphorylated 53BP1 remains excluded from chromatin until cells progress into G1 phase [7]. Based on these findings, it was hypothesized that mitosis-specific PTMs on RNF8 and 53BP1 might preclude formation of repair-competent IRIF [7]. However, the precise mechanistic explanation of the "interrupted" DDR in mitosis remained to be unravelled.

A recent study published in Science by the group of Daniel Durocher addressed the question of how full IRIF assembly and DSB repair are prevented in mitotic cells [8]. First, Orthwein et al. focused on the mechanism that abrogates RNF8 recruitment to DSBs during mitosis. They demonstrated that CDK1-dependent mitosis-specific phosphorylation of RNF8 on T198 abol- ished interaction between RNF8 and its target phospho-TQXF motifs in MDC1. This important finding was somewhat surprising, given that $\mathrm{MDC} 1$ binding is mediated by the RNF8 FHA domain [5, 6] and T198 is located some distance away from this domain. It will thus be interesting to see how T198 phosphorylation abrogates MDC1 binding, for example via T198 being juxtaposed to the FHA domain in the RNF8 3D structure, through phosphorylated T198 docking with the phospho-binding region of the FHA domain, or via another mechanism. In this regard, we note that T198 is part of an STP motif, which upon modification by CDK1 could constitute a priming site for PLK1 kinase [9]. Thus, T198 phosphorylation might be followed by PLK1-mediated RNF8 phosphorylation. Interestingly, certain sites in RNF8 conform to the PLK1 consensus motif, with those at T39 and T316 being evolutionarily conserved in vertebrates. Moreover, T39 is located in the FHA domain, close to R42, mutation of which abolishes RNF8 interaction with MDC1 $[5,6]$. It would therefore be worthwhile mutating these potential PLK1 sites and establishing whether this affects mitotic control of RNF8 binding to MDC1.

After identifying T198 as critical for preventing RNF8 recruitment to DSBs during mitosis, Orthwein et al. observed that, while mutating this residue to alanine restored recruitment of RNF8 (and BRCA1) to mitotic IRIF, 53BP1 still remained excluded from DSB sites. This prompted the authors to look for mitosisspecific PTMs of 53BP1 by mass spectrometry, leading to the discovery of two novel phosphosites mapped to the recently described ubiquitin-dependent 
recruitment (UDR) motif, which mediates binding to ubiquitylated $\mathrm{H} 2 \mathrm{~A}$ and is required for 53BP1 IRIF formation [10]. Notably, the same residues, T1609 and S1618, were also identified by Chowdhury and colleagues [11] as target sites for the PP4C/R3 $\beta$ phosphatase. This group showed that T1609 and S1618 must be dephosphorylated for 53BP1 to form IRIF. In accord with these findings, Orthwein et al. established that when T1609 and S1618 were mutated to alanines, the ensuing "53BP1-TASA" protein was recruited to sites of DNA damage during mitosis in cells expressing RNF8-T198A. Moreover, unlike normal cells, cells co-expressing RNF8T198A and 53BP1-TASA carried out DSB joining reactions during mitosis and were extremely hypersensitive to ionizing radiation (IR). The authors also found that, following irradiation in mitosis, cells carrying these mutant RNF8 and 53BP1 proteins displayed increased rates of kinetochore-positive micronucleus formation, suggesting mis-segregation of full chromosomes. In addition, chromosomes in these cells were prone to sister telomere fusions, thereby helping to explain their elevated levels of aneuploidy and IR hypersensitivity.

The research described above has not only revealed how DSB repair is suppressed in mitosis but has also established that this suppression is biologically important. Orthwein et al. propose that, as mitotic telomeres become "underprotected" when mitosis is prolonged upon stress [12], this could lead to telomere fusion if DNA end-joining pathway is active. The suppression of DSB signaling and repair mediated by RNF8 and 53BP1 mitotic phosphorylation therefore probably evolved as a mechanism to mitigate this threat to genome stability. A key question that still remains is why mitotic telomeres become underprotected in the first place? Also, what features in telomere structure or replication and segregation processes make it more beneficial for the cells to keep chromosome ends less protected at the cost of inhibiting the DDR during mitosis? Finally, given that cancers often harbor cell cycle and/or DDR defects [1], it will be of interest to see whether defective mitotic control of DSB repair might play a role in tumor evolution, or could provide opportunities for developing better anti-cancer therapies.
Rimma Belotserkovskaya ${ }^{1}$, Stephen P Jackson ${ }^{1,2}$

${ }^{1}$ The Wellcome Trust and Cancer Research UK Gurdon Institute and Department of Biochemistry, University of Cambridge, Cambridge, CB2 $1 Q N$, England, UK; ${ }^{2}$ The Wellcome Trust Sanger Institute, CB10 1SA Hinxton, Cambridge, UK

Correspondence: Stephen P Jackson ${ }^{\mathrm{a}}$, Rimma Belotserkovskaya ${ }^{\mathrm{b}}$

aE-mail: s.jackson@gurdon.cam.ac.uk

bE-mail: r.belotserkovskaya@gurdon.cam.ac.uk

\section{References}

1 Jackson SP, Bartek J. Nature 2009; 461:10711078.

2 Palm W, de Lange T. Annu Rev Genet 2008; 42: 301-334.

3 Bekker-Jensen S, Mailand N. DNA Repair (Amst) 2010; 9:1219-1228.

4 Polo SE, Jackson SP. Genes Dev 2011; 25:409-433.

5 Kolas NK, Chapman JR, Nakada S, et al. Science 2007; 318:1637-1640.

6 Mailand N, Bekker-Jensen S, Faustrup H, et al. Cell 2007; 131:887-900.

7 Giunta S, Belotserkovskaya R, Jackson SP. $J$ Cell Biol 2010; 190:197-207.

8 Orthwein A, Fradet-Turcotte A, Noordermeer SM, et al. Science 2014; 344:189-193.

9 Elia AE, Rellos P, Haire LF, et al. Cell 2003 115:83-95.

10 Fradet-Turcotte A, Canny MD, EscribanoDíaz C, et al. Nature 2013; 499:50-54.

11 Lee DH, Acharya SS, Kwon M, et al. Mol Cell 2014; 54:512-525.

12 Hayashi MT, Cesare AJ, Fitzpatrick JA. Nat Struct Mol Biol 2012; 19:387-394. 